\title{
ADEQUATE SALT INTAKE ATTENUATES MINERALOCORTICOID RECEPTOR ANTAGONIST-INDUCED HYPERKALEMIA IN PATIENTS WITH PRIMARY ALDOSTERONISM.
}

\author{
Fountoulakis Stelios, Papanastasiou Labrini \& Piaditis George \\ Clinic of Endocrinology and Diabetes Center, General Hospital of Athens 'G. Gennimatas', Athens, Greece
}

Introduction and objectives: Mild hyperkalemia is a common side-effect of mineralocorticoid receptor antagonists (MRA), which can be precipitated by minimizing dietary salt intake. Restoration of adequate salt intake could overcome diminished kaliuresis and restore potassium plasma levels. Aim of this study was the evaluation of the effect of short-term adequate salt consumption on plasma potassium levels in relation to the mean, maximum and minimum blood pressure (BP) and weight in MRA-treated sodium-depleted hyperkalemic patients with primary hyperaldosteronism (PA)

Description of methods/Design: Nine MRA-treated sodium-depleted PA patients $(67.7 \pm 9.7$ years of age, 7 females-2 males) were recruited. BP was documented twice daily by the patients and renin (plasma), aldosterone (plasma), potassium and sodium (plasma and 24hour urine) levels were measured while patients were following a sodium-restricted diet and after one month of adequate dietary salt supplementation ( $4 \mathrm{~g}$ of salt/day). Body weight and waist circumference were also documented. Exclusion criteria were: renal or hepatic failure, malabsorptive diseases of the gastroenteric tract, uncontrolled thyroid disease, malignancy, medications interfering with $\mathrm{Na}+/ \mathrm{K}+$ homeostasis (NSAIDs, diuretics, b-blockers, steroids, insulin, ACE-i, ARBs).

\begin{tabular}{ll|}
\hline Weight $(\mathrm{Kg})$ & $78.2 \pm 21.6$ \\
\hline Age $($ years $)$ & $67.7 \pm 9.7$ \\
Sex $(\mathrm{n})$ & 7 females- 2 males \\
Treatment $(\mathrm{n})$ & $\begin{array}{l}\text { Spironolactone }(50-150 \mathrm{mg})=6 \\
\text { Eplerenone }(50 \mathrm{mg})=3 \\
* 1 \text { patient }=\text { plus calcium channel blocker }\end{array}$ \\
& Bilateral cause $=6$ \\
PA $(\mathrm{n})$ & Unilateral cause $=3$
\end{tabular}

Results: Salt supplementation (24hour urine sodium: $199.39 \pm 50.46$ vs $101.06 \pm 41.78 \mathrm{mmol} / \mathrm{d}$ ) increased kaliuresis $(68.9 \pm 21.7$ vs $54.21 \pm 17.6 \mathrm{mmol} / \mathrm{d}, \mathrm{p}<0.001)$ and resulted in a statistically significant decrease of potassium $(4.64 \pm 0.34$ vs $5.28 \pm 0.26 \mathrm{mmol} / \mathrm{Lt}, \mathrm{p}<0.001)$, renin and aldosterone levels, without affecting plasma sodium levels, mean diastolic or systolic BP $(128.3 \pm 10.6$ vs $129.2 \pm 10.2$ and $71 \pm 7.6$ vs $71.1 \pm 7.5 \mathrm{mmHg}$, respectively) or minimum and maximum BP values. Limitations of the study included: small patient number and short-term follow-up.

$\begin{array}{llll} & \text { Prior to salt } & \text { After salt } & \text { P } \\ \mathrm{K}(\mathrm{mmol} / \mathrm{Lt}) & 5.28 \pm 0.26 & 4.64 \pm 0.34 & <0.001 \\ \mathrm{Na}(\mathrm{mmol} / \mathrm{Lt}) & 138 \pm 2.69 & 138.4 \pm 2.65 & \mathrm{NS} \\ \mathrm{Kur}(\mathrm{mmol} / \mathrm{d}) & 54.21 \pm 17.6 & 68.9 \pm 21.7 & <0.001 \\ \mathrm{Naur}(\mathrm{mmol} / \mathrm{d}) & 101.06 \pm 41.78 & 199.39 \pm 50.46 & <0.001 \\ \text { Renin }(\mathrm{pg} / \mathrm{ml}) & 53 \pm 48.5 & 43.2 \pm 47 & 0.004 \\ \text { Aldosterone }(\mathrm{pmol} / \mathrm{Lt}) & 2435 \pm 1667 & 1603 \pm 1670 & 0.015\end{array}$

\begin{tabular}{l|l|l|l|}
\hline & Prior to salt & After salt & P \\
\hline SBP $(\mathrm{mmHg})$ & $129.2 \pm 10.2$ & $128.3 \pm 10.6$ & NS \\
DBP $(\mathrm{mmHg})$ & $71.1 \pm 7.5$ & $71 \pm 7.6$ & NS \\
SBPmin $(\mathrm{mmHg})$ & $115.3 \pm 13.5$ & $114 \pm 12.6$ & NS \\
DBPmin $(\mathrm{mmHg})$ & $62.6 \pm 10.5$ & $62.1 \pm 10.1$ & NS \\
SBPmax $(\mathrm{mmHg})$ & $148 \pm 7.9$ & $150.7 \pm 8.3$ & NS \\
DBPmax $(\mathrm{mmHg})$ & $80.7 \pm 7.5$ & $82.4 \pm 7.4$ & NS \\
Weight $(\mathrm{kg})$ & $78.2 \pm 21.6$ & $78.1 \pm 21.3$ & NS \\
Waist $(\mathrm{cm})$ & $105.6 \pm 19.5$ & $105.7 \pm 19.5$ & NS
\end{tabular}

Conclusion: Adequate salt intake can attenuate MRA-induced hyperkalemia in sodium-depleted PA patients without short-term effects on BP. The underlying mechanism could involve restoration of adequate $\mathrm{Na} / \mathrm{K}$ exchange ( $\mathrm{Na/K}$ ATPase) and/or translocation of sodium absorption from the convoluted tubule, where sodiumchloride symporter (NCC) is prevalent, to the collecting tubule where epithelial sodium channel (ENaC) and renal outer medullary potassium channel (ROMK) dominate, in the face of blocked aldosterone receptor.

Posterser 